\title{
The e-ASTROGAM space mission for MeV-GeV gamma-ray and multi-messenger astrophysics
}

\section{Stefano Ciprini*}

Istituto Nazionale di Fisica Nucleare (INFN), Sezione di Roma Tor Vergata, I-00133, Roma, Italy

Space Science Data Center, Agenzia Spaziale Italiana (SSDC ASI), I-00133, Roma, Italy

E-mail: stefano.cipriniessdc.asi.it

\section{Alessandro De Angelis}

Dipartimento di Fisica e Astronomia Università di Padova and Istituto Nazionale di Fisica Nucleare (INFN), Sezione di Padova, I-35131, Padova, Italy

Dipartimento di Scienze Mat., Inf. e Fis. Università di Udine, I-33100 Udine, Italy

Laboratório de Instrum . e Física Exper. de Partículas (LIP/IST), PT-1649-003 Lisboa, Portugal

\section{Vincent Tatischeff}

Centre de Sciences Nucléaires et de Sciences de la Matière (CSNSM), Centre Nationale de la Recherche Scientifique (CNRS), and Université Paris-Sud, F-91405, Orsay, France

Future high-energy, multi-frequency survey astronomy, time-domain and multi-parameters inference, multimessenger astro-particle physics, will not be able to grow in a really comprehensive, synergetic and serendipitous way, if an important, broad, electromagnetic window in the Universe, where interesting and multiple physical processes occur, is left unobserved. In this view e-ASTROGAM is a project of breakthrough space observatory mission, with large international interest, characterized by a detector composed by a silicon tracker, a calorimeter, and an anticoincidence system, dedicated to the, so called, poorly-known MeV-energy sky, through the detection of photons from about $150 \mathrm{keV}$ to $3 \mathrm{GeV}$ energy. e-ASTROGAM is optimized for simultaneous detection of Compton and pair-producing photon events, with optimal capabilities in gammaray continuum, nuclear line spectroscopy, spatial imaging, gamma-ray polarization measurement, and with a large field of view, essential for survey/time-domain multi-messenger synergy. All these capabilities, will be able to open a rich, interdisciplinary, science menu in a electromagnetic band, the MeV Universe, probed only in a shallow way by previous satellites. e-ASTROGAM will be the ground-breaking instrument in case of nearby (about $<20 \mathrm{Mpc}$ ) supernovae and kilonovae events, directly probing nucleosynthesis processes with a MeV line sensitivity one to two orders of magnitude better than previous/current instruments. The origin of key nuclear isotopes, responsible for the creation of $\sim 50 \%$ the abundances of the nuclei heavier than iron, and for radioactive/chemical evolution of our Galaxy will be accurately determined. e-ASTROGAM is also a general-purpose satellite for broad and different scientific communities, with a rich scientific potential, placed in the enormous spectral energy vacuum between the multitude of soft-X-ray satellites (Athena for example) and the ( $\gtrsim 40 \mathrm{GeV})$ band of the Cherenkov Telescope Array (CTA). Powerful, multi-messenger experiments such as LISA, LIGO, Virgo, KAGRA, Einstein Telescope, Cosmic Explorer, IceCube-gen2, KM3NeT, SKA, ALMA, JWST, E-ELT, LSST, Athena, CTA will have a consistent leap in science results if coupled with e-ASTROGAM or similar all-sky/wide survey MeV space telescopes.

Accretion Processes in Cosmic Sources - II - APCS2018

3-8 September 2018

Saint Petersburg, Russian Federation

\footnotetext{
* Speaker.
} 


\section{Introduction}

The worst-covered part of the electromagnetic spectrum, superficially observed with nonsufficient sensitivity and a poor sky coverage, is the wide band between $0.1 \mathrm{MeV}$ and $100 \mathrm{MeV}$. Many astrophysical objects, physical ambient and systems have the peak of their electromagnetic emissivity in this energy range. For example gamma-ray bursts, GRBs, a a large fraction of blazars and radiogalaxies, pulsars, gamma-ray binaries, novae and other astrophysical objects. In particular the $\mathrm{MeV}$ photon energy range (medium gamma rays) is the domain of nuclear gamma-ray lines and nucleosynthesis, representing for nuclear astrophysics what optical astronomy is for atomic astrophysics. In addition, after almost a century, the unsolved dark matter mystery, after huge WIMP searches and efforts, is now better addressing to low-energy species from $\mathrm{GeV}$ down to feV masses, with several hidden-sectors and relics models with masses in the $\mathrm{keV}-\mathrm{MeV}$ range.

In general, scientific challenges and expected discovery spaces for modern astronomy can be summarized as the following: early Universe primordial objects and stars in the Universe and cosmology; large-scale structure of the Universe and multiverse; nature of dark matter and nature of dark energy; origin of cosmic rays and physics of particle acceleration; compact objects and energetic, violent phenomena like black holes (BHs), neutron stars (NSs), accretion processes, outflows and relativistic jets, BH/NS mergers; BHs and dark mater interplay; search for new and exotic objects like monopoles, quark stars and other; high-energy production sites and processes; exoplanets, solar system bodies exploration; astrobiology, the search for life and also the search for technosignatures by exo-civilizations in the Universe; Sun-Earth interactions and physics of the heliosphere and the Sun. For most of these hot science topics is important to understand the creation and evolution of nuclei and chemical elements in our solar neighborhood, our Galaxy, and in the extragalactic space ${ }^{1}$.

e-ASTROGAM $[8,9,18,19]$ (and references therein) is a project for a breakthrough medium/high energy gamma-ray space observatory (MeV-energy space telescope concept), operating in a photon energy band where the sky is still poorly explored, and proposed for 2030s, a mature epoch of multi-messenger astrophysics and astro-particle physics when entirely new and powerful synergies will be possible. This $\mathrm{MeV}$ space telescope mission will provide unique and complementary gamma-ray data of significant interest to broad and different astronomical and astro-particle physics communities, in a decade when powerful large-scale ground-based (and under-water/ice/ground) experiments, and ground based and space borne observatories for multi-messenger astronomy will be operational for the detection of gravitational waves, neutrinos, ultra-high-energy cosmic rays (UHECRs) and multi-wavelength electromagnetic radiation. Without a space telescope like eASTROGAM in orbit, the power of future multi-wavelength and multi-messenger synergy and scientific serendipity, will be depressed hindering potential important discoveries. This is particularly true in case of nearby supernovae or kilonovae explosions, because a $\mathrm{MeV}$ space observatory like e-ASTROGAM will represent the ground-breaking instrument able to directly probe nucleosynthesis, with the identification of the origin of key nuclear isotopes [7, 10], thought to be responsible for the creation of approximately half the abundances of the nuclei heavier than iron, and shedding

1“The formation of stars and planets, and the development of the chemistry of life, can be understood only in the context of the creation and evolution of elements" - S.Boggs (in The Advanced Compton Telescope project, 2006) 

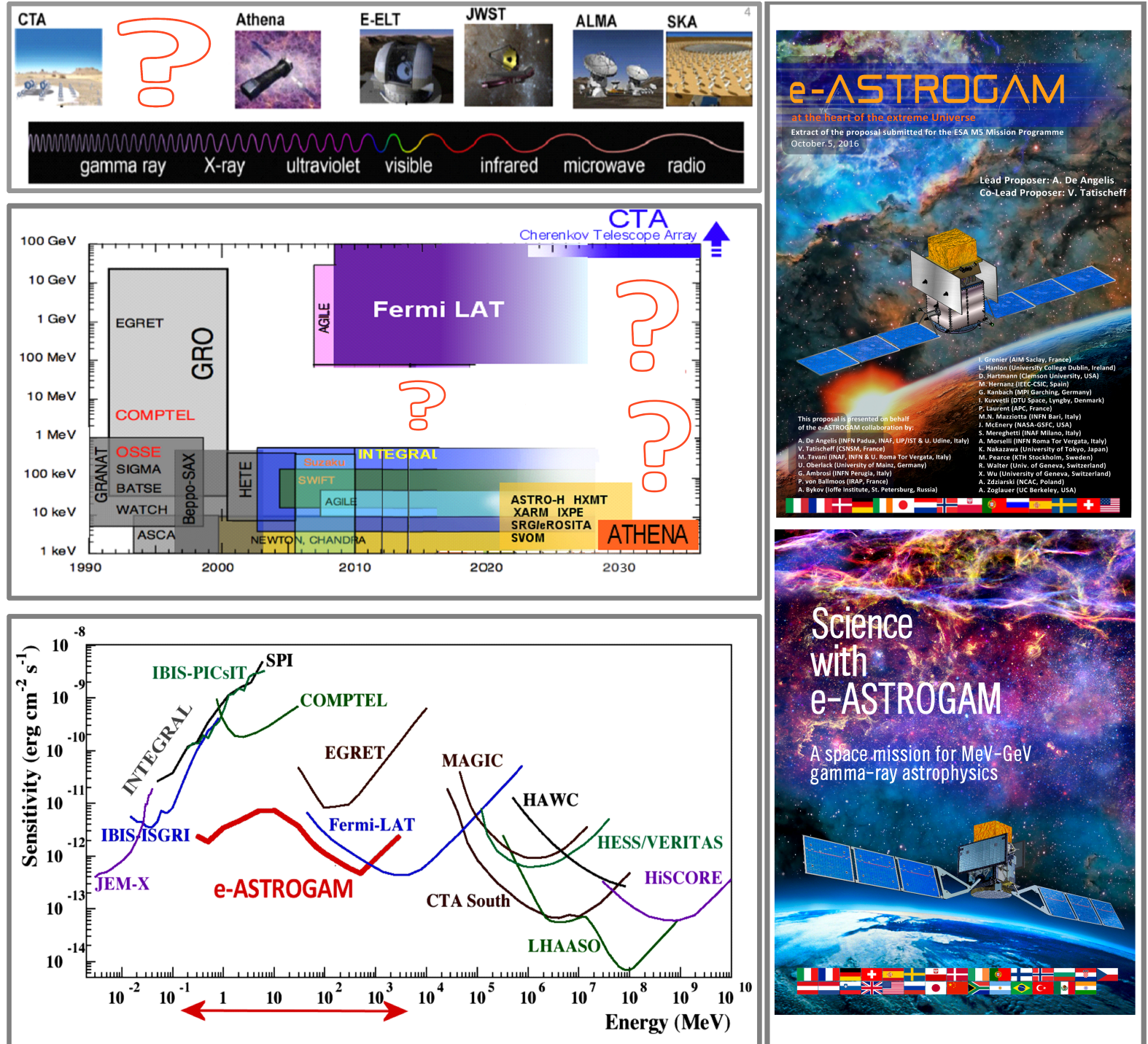

Figure 1: Left upper and central panels: there is a particularly broad and crucial spectral energy electromagnetic range that is continuing to be unobserved and ignored by space agencies since about 1999 after the Compton Gamma Ray Observatory (CGRO) COMPTEL instrument. This vacuum of information is an important problem of 2020s and 2030s for multifrequency and multimessenger astronomy and astro-particle physics. The situation will be dramatic after the Fermi LAT mission, leaving the sky unobserved from photon energies of some $\mathrm{keV}$ (Athena) to about $>40 \mathrm{GeV}$ of CTA. Left lower panel: point source continuum differential sensitivity of different X- and gamma-ray instruments. The curves for INTEGRAL/JEM-X, IBIS (ISGRI and PICsIT), and SPI are for an effective observation time $T_{\mathrm{obs}}=1 \mathrm{Ms}$. The COMPTEL and EGRET sensitivities are given for the typical observation time accumulated during the $\sim 9$ years of the CGRO mission. The Fermi LAT sensitivity is for a high Galactic latitude source in 10 years of observation in survey mode. For MAGIC, VERITAS (sensitivity of HESS is similar), and CTA, the sensitivities are given for $T_{\mathrm{obs}}=50$ hours. For HAWC $T_{\mathrm{obs}}=5 \mathrm{yr}$, for LHAASO $T_{\mathrm{obs}}=1 \mathrm{yr}$, and for HiSCORE $T_{\mathrm{obs}}=1000 \mathrm{~h}$. The e-ASTROGAM sensitivity is calculated at $3 \sigma$ for an effective exposure of 1 year and for a source at high Galactic latitude. Adapted from [18]. Right panels: covers of the e-ASTROGAM Mission Proposal [9] and the science White Book [8].

light on the radioactive nuclear species and, in general, on cosmo-chemistry and evolution of our Milky Way Galaxy. 


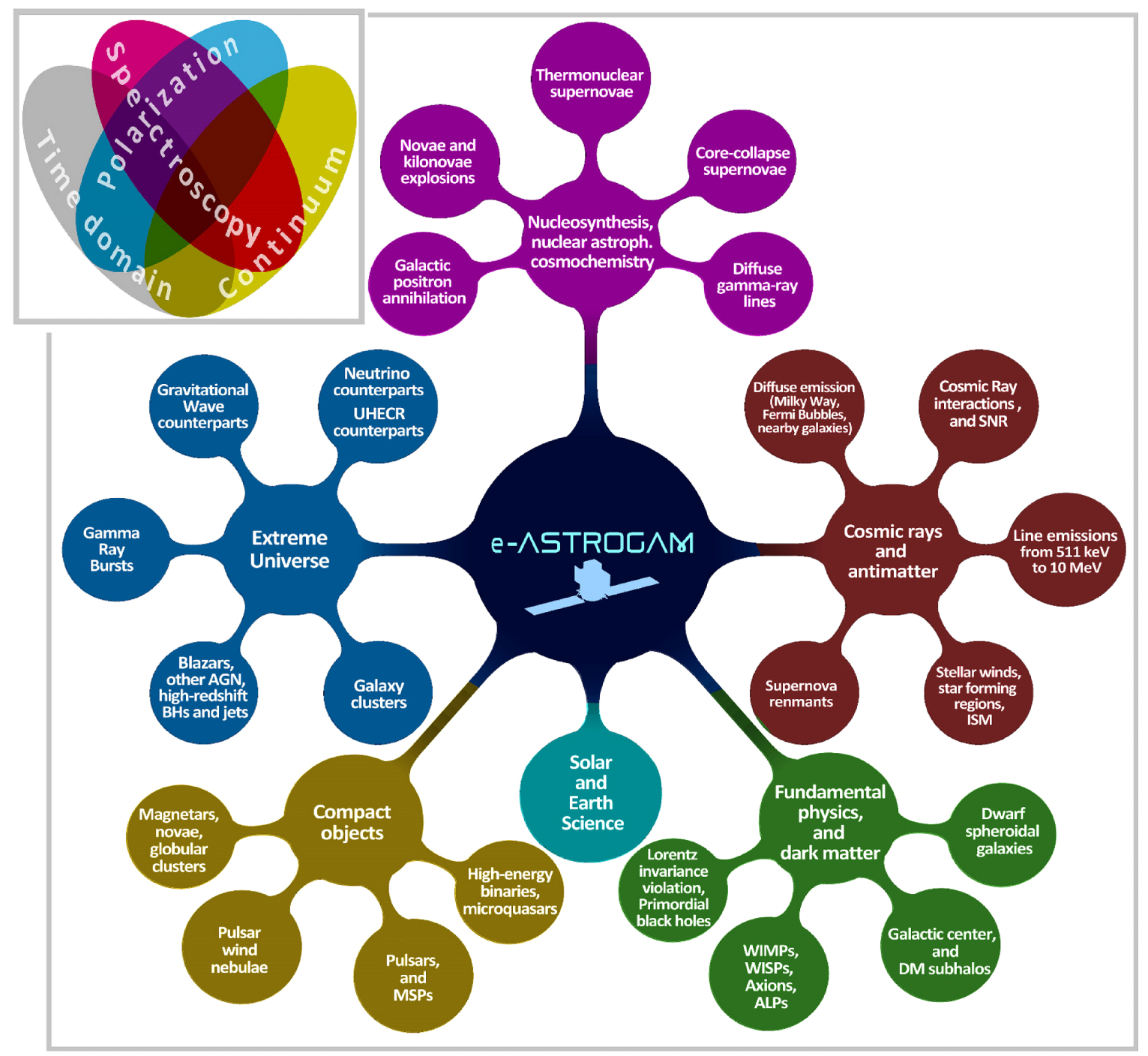

Figure 2: e-ASTROGAM has unprecedented capabilities in $\mathrm{MeV}$ gamma-ray continuum spatial imaging and spectroscopy, nuclear line spectroscopy and line imaging, gamma-ray polarization measurement and large field of view, the latter meaning effective spatial sky survey and time domain monitor. An example of science visualization for e-ASTROGAM is represented here in a tentative scheme for its research topics (adapted from [8]). This multitude of topics and astrophysical targets reflects the general-purpose character of the mission, serving broad and diverse science communities, beyond the three major science core topics that are identifying the e-ASTROGAM mission: 1) the HE and nuclear processes at the heart of the extreme Universe with prospects for the 2030s multi-frequency and multi-messenger science; 2) the origin and impact of high-energy particles on galaxy evolution, from cosmic rays to antimatter; 3) the nucleosynthesis and the chemical enrichment of our Milky Way Galaxy.

The e-ASTROGAM instrument $[8,9,18,19]$ is composed of a silicon tracker detector, a calorimeter and an anticoincidence system, dedicated to the observation of the electromagnetic sky in photon energies from about $150 \mathrm{keV}$ to $3 \mathrm{GeV}$. The lower energy limit can be pushed as low as $30 \mathrm{keV}$ for gamma-ray burst detection and very bright transients with pure calorimeter detection. e-ASTROGAM is optimized for simultaneous detection of Compton and pair-producing photon events with a single instrument over such a wide energy band, dramatically improving the COMPTEL, INTEGRAL and Fermi LAT sensitivities. This satellite is able to make pioneering ob- 
servations of the most powerful Galactic and extragalactic sources, elucidating the nature of their accretion processes, particle acceleration, relativistic outflows and their effects on the surrounding ambient and nucleosynthesis, with unprecedented capabilities in $\mathrm{MeV}$ gamma-ray continuum, nuclear line spectroscopy, spatial imaging, polarization measurement and with a large field of view. e-ASTROGAM can be considered a general-purpose space mission for the observation of the lowand high-energy (sub-MeV to GeV energy) gamma-ray sky, serving broad and different scientific communities, therefore with a high productivity in term of scientific results and publications. It is devoted to fill a pivotal and large gap in the electromagnetic spectrum, left empty by the currently planned instruments, a gap between the multitude of current (and the next) soft-X-ray satellites (Athena in particular) observing up to a dozen of $\mathrm{keV}$, and the Cherenkov Telescope Array (CTA) observing the sky above about $40 \mathrm{GeV}$ (Fig. 1). On the other hand e-ASTROGAM can be also viewed as a nucleosynthesis space telescope for supernovae, kilonovae and novae, devoted to understand the creation of key isotopes in such transients, and the enrichment and evolution of our Galaxy, also in terms of cosmic rays and antimatter content.

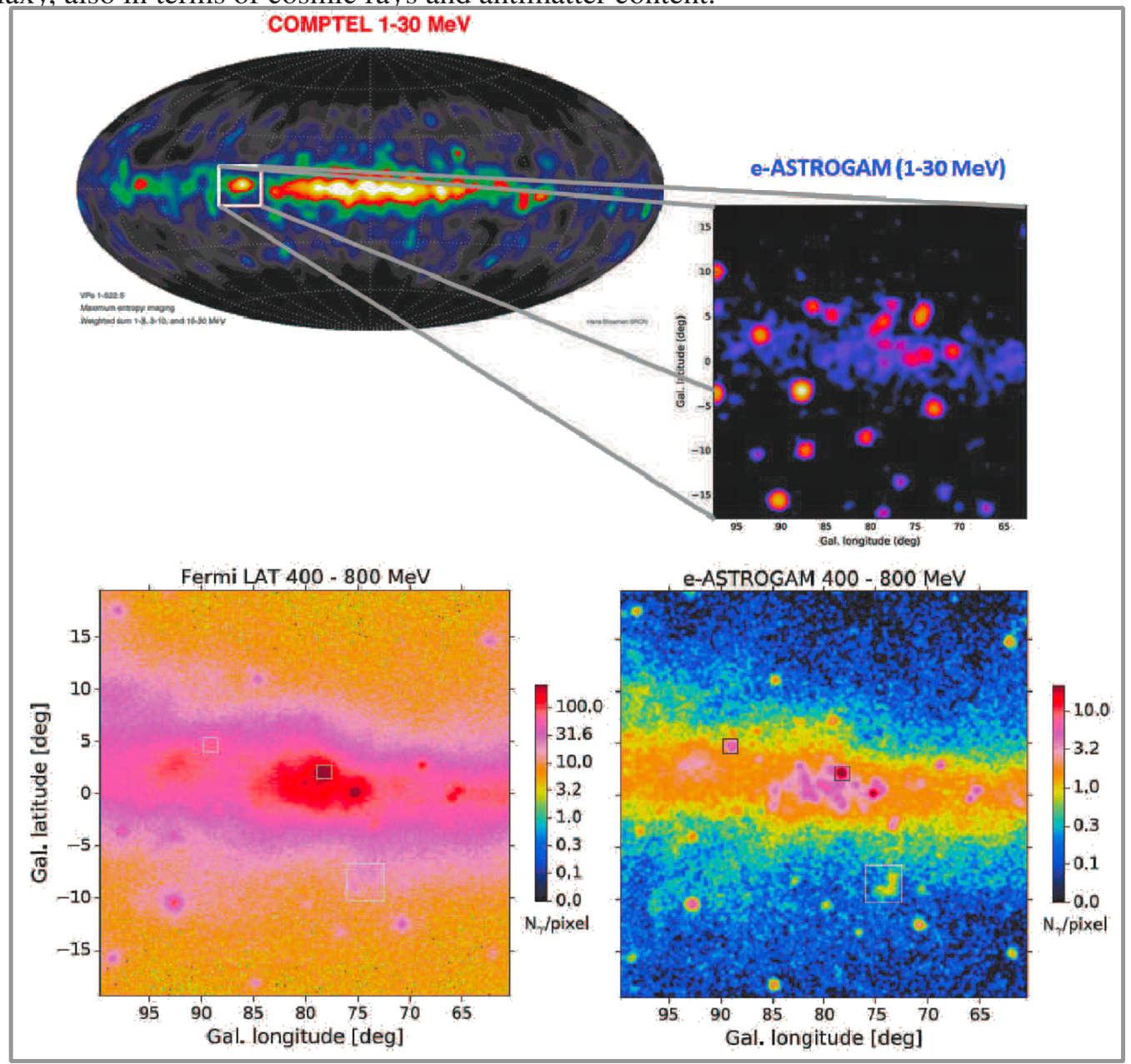

Figure 3: An example of the capability of e-ASTROGAM to transform our knowledge of the MeV-GeV sky. Upper panel: the upper left figure shows the 1-30 MeV sky as observed by COMPTEL in the 1990s and the upper right figure shows the simulated Cygnus region in the 1-30 MeV energy region from e-ASTROGAM. Lower panel: comparison between the view of the Cygnus region by Fermi LAT in 8 years (left) and that by e-ASTROGAM in one year of effective exposure (right) between 400 and $800 \mathrm{MeV}$. 


\section{Threefold core science topic}

Even considering the general-purpose character of e-ASTROGAM, provided mainly by its large field of view and the many secondary and ancillary data and targets, the mission is well defined and identified by three core science topics composing, motivating of the mission [8, 18]: 1) High energy (HE) and nuclear processes at the heart of the extreme Universe with prospects for the 2030s multi-frequency/multi-messenger science; 2) the origin and impact of high-energy particles on galaxy evolution, from cosmic rays to antimatter; 3 ) the nucleosynthesis and the chemical enrichment of our Galaxy.

\subsection{Processes at the heart of the extreme Universe: prospects for the multi-messenger astronomy of the 2030s}

The $\mathrm{MeV}$ and $\mathrm{GeV}$ gamma-ray band is crucial to understand the most violent and extreme astrophysical processes, specifically to understand sources with accretion, relativistic jet and outflow phenomena, both Galactic like novae, kilonovae, supernovae, microquasars, X-ray binaries, Wolf-Rayet stars, young stellar objects associations and star forming regions, pulsars, magnetars, soft X-ray/gamma-ray repeaters, supernova renmants, and extragalactic, like active galactic nuclei (AGN), galaxy clusters and other sources. The MeV energy regime is placed at the thermal and non-thermal processes boundary and interplay region, and at the transition region from the low energy continuum to the high-energy emission component dominated by, poorly understood, particle acceleration processes. A substantial fraction of the radiated power is emitted in the $\mathrm{MeV}$ band, and the unprecedented sensitivity of e-ASTROGAM in this energy range (Fig. 1) will allow us an unprecedented view of the high-energy processes in action close to supermassive black holes (SMBHs) or to star-sized BHs (in our Galaxy or in nearby galaxies), in addition to novel views of powerful explosions and transients like gamma-ray bursts (GRBs) and mergers of two NSs or of a NS and a BH.

e-ASTROGAM is able to determine the composition (hadronic or leptonic) and structure of outflows and jets, which strongly influences the environment, thanks to breakthrough gamma-ray polarimetric and spectroscopy capabilities, and to identify acceleration processes (diffusive shocks, magnetic reconnection, plasma effects and other possibilities) leading to different particle energy distributions. It can also shed light on the role of the magnetic field in powering ultra-relativistic jets in GRBs. MeV observations are able to impact on multi-messenger astro-particle physics: gravitational-wave (GW) and gamma-ray observations of high-energy transients are complementary and essential to obtain a more complete identification and knowledge of the sources and progenitors; gamma-ray observations also play a decisive role for our understanding of the astrophysical origin and sources of very/extreme high energy neutrinos and UHECRs.

\subsection{The origin and impact of cosmic-ray particles on galaxy evolution}

e-ASTROGAM is able to solve the outstanding issue of the origin and propagation of lowenergy cosmic rays in the interstellar medium (ISM) of our Milky Way Galaxy. It will measure cosmic-ray diffusion in interstellar clouds and their impact on gas dynamics and state providing, therefore, crucial diagnostics about the cosmic-ray feedback on star formation, ISM structures, 
galactic winds and outflows, as well as about the growth of interstellar magnetic field. The improved sensitivity and angular resolution of e-ASTROGAM (Fig. 4) will be important to probe the interplay between cosmic rays and the turbulent medium of star forming regions (e.g., Cygnus Cocoon) during the early steps of their voyage across the Galaxy. Young supernova remnants (SNRs) as potential cosmic-ray sources can be observed by e-ASTROGAM with enough sensitivity, allowing to distinguish the emission produced by the interactions of cosmic-ray nuclei with the ambient gas and the non-thermal emission from cosmic-ray electrons. Multi-frequency observations of eASTROGAM joined with high-resolution radio-band and X-ray spatial structure observations, will provide unique information on cosmic-ray injection into the acceleration region, on the structure of magnetic fields and the spectrum of fresh cosmic rays released into surrounding clouds of the SNRs.

\subsection{Nucleosynthesis and the chemical enrichment of our Galaxy}

The e-ASTROGAM gamma-ray line sensitivity is more than an order of magnitude better than that of previous or current instruments. The deep exposure of the Galactic plane region will determine how different isotopes $\left({ }^{26} \mathrm{Al},{ }^{44} \mathrm{Ti},{ }^{60} \mathrm{Fe}\right)$ are created in stars and distributed in the interstellar medium. e-ASTROGAM will also unveil the recent history of supernova explosions in our Galaxy and detect the radioactive emission of Galactic classical novae $\left({ }^{7} \mathrm{Be},{ }^{22} \mathrm{Na}, 511 \mathrm{keV}\right.$ line $)$ for the first time. e-ASTROGAM will also detect a significant number of supernovae in nearby galaxies, thus addressing fundamental issues in the explosion mechanisms of both core-collapse and thermonuclear supernovae. The gamma-ray line data $\left({ }^{56} \mathrm{Ni},{ }^{56} \mathrm{Co}\right)$ will provide a much better understanding of Type Ia supernovae [7, 10] and their evolution with look-back time and metallicity, which is a pre-requisite for their use as standard candles for precision cosmology.

\subsubsection{Novae explosions}

Accreting white dwarfs in close binary systems can explode as novae and/or as SNe Ia. Novae are responsible for the enrichment of the Galaxy in some species and for the peculiar isotopic signatures found in some pre-solar grains. Understanding the origin of the elements in the Galaxy and in the whole Universe is an important topic, intimately related to explosive nucleosynthesis and emission of gamma-rays that directly trace isotopes. Nova ejecta are enriched in CNO nuclei, as well as in $\mathrm{Ne}, \mathrm{Na}, \mathrm{Mg}$ and even $\mathrm{S}$ in some cases. They also produce ${ }^{7} \mathrm{Be}$, which through electron-capture becomes ${ }^{7} \mathrm{Li}$; the role of novae in the origin of ${ }^{7} \mathrm{Li}$ in the Galaxy and the Universe is a hot scientific topic. Also the contribution of novae to the galactic content of ${ }^{26} \mathrm{Al}$, traced by $1.809 \mathrm{MeV}$ gamma-ray line detected since long ago, is still not well known. There are two main types of binary systems where white dwarfs can accrete matter and subsequently explode as novae: the most common case of a cataclysmic variable where the companion is a main sequence star transferring $\mathrm{H}$-rich matter, and the case of a white dwarf that can explode as a nova is a symbiotic

\begin{tabular}{lllll}
\hline Isotope & Lifetime & Main process & Type of emission & Nova type \\
\hline${ }^{13} \mathrm{~N}$ & $862 \mathrm{~s}$ & $\beta^{+}$-decay & $511 \mathrm{keV}$ line and continuum & CO and ONe \\
${ }^{18} \mathrm{~F}$ & $158 \mathrm{~min}$ & $\beta^{+}$-decay & $511 \mathrm{keV}$ line and continuum & CO and ONe \\
${ }^{7} \mathrm{Be}$ & 77 days & $e^{-}$-capture & $478 \mathrm{keV}$ line & CO \\
${ }^{22} \mathrm{Na}$ & 3.75 years & $\beta^{+}$-decay & 1275 and $511 \mathrm{keV}$ lines & ONe \\
${ }^{26} \mathrm{Al}$ & $10^{6}$ years & $\beta^{+}$-decay & 1809 and $511 \mathrm{keV}$ lines & ONe \\
\hline
\end{tabular}

Figure 4: List of main radioactive isotopes in nova ejecta. 


\begin{tabular}{|l|l|l|}
\hline Source type & Source counts (3 years) & New sources \\
\hline \hline Total & $3000-4000$ & $\sim 1900$ (including GRBs) \\
Galactic & $\sim 1000$ & $\sim 400$ \\
MeV blazars & $\sim 350$ & $\sim 350$ \\
GeV blazars & $1000-1500$ & $\sim 350$ \\
Other AGN $(<10 \mathrm{MeV})$ & $70-100$ & $35-50$ \\
Supernovae & $10-15$ & $10-15$ \\
Classical novae & $4-6$ & $4-6$ \\
Long GRBs & $\sim 540$ & $\sim 540$ \\
Short GRBs & $\sim 180$ & $\sim 180$ \\
\hline
\end{tabular}

Table 1: Estimated number of sources of various classes detectable by e-ASTROGAM in three years. The last column gives the number of sources not known before in any electromagnetic wavelength.

binary where the white dwarf accretes H-rich matter from the stellar wind of a red giant companion. Soft and high-energy gamma rays emitted by novae have two very different origins: radioactivity and high energy particle accelerated in diffusive shocks. With a sensitivity of e-ASTROGAM for the nova broad lines at $1.275 \mathrm{MeV}$ and $478 \mathrm{keV}>25$ ( $>13$ for $478 \mathrm{keV}$ ) times better than that of INTEGRAL SPI, detectability distances would be 5 (3.6) times deeper, reaching $3 \mathrm{kpc}$ and 2 $\mathrm{kpc}$, meaning it could be expected to detect one nova per year [8]. In addition to the direct and unambiguous detection for the first time of the radioactive nuclei ${ }^{22} \mathrm{Na}$ and ${ }^{7} \mathrm{Be}-{ }^{7} \mathrm{Li}$ in novae eASTROGAM observations would help to answer some key questions about nova explosions, and their accretion processes.

\subsection{Source counts}

Beyond the addressing of its core scientific goals, e-ASTROGAM will be able to achieve many serendipitous discoveries through its combination of wide field of view and improved sensitivity, measuring in three or six (extended) years the spectral energy distributions of thousands of Galactic and extragalactic sources (Fig. 4 and Table 1). An extensive review of the science achievable with this mission is given in the official e-ASTROGAM White Book [8]. Table 1 summarizes conservative estimates of the number of sources detectable in the first three years, based on current knowledge and $\log N-\log S$ determinations of Galactic and extragalactic sources, including GRBs. Noteworthy, the 4th Fermi LAT catalog [4] contains more than 1900 unidentified sources in the $>100 \mathrm{MeV}$ energy range with no counterparts at other wavelength and most of them will be detected by e-ASTROGAM, in addition to a relevant number of new unidentified sources. The discovery domain of e-ASTROGAM for new sources and source classes is expected to be very large and interesting.

An important characteristic of e-ASTROGAM is its ability to measure polarization in the $\mathrm{MeV}$ energy range, which is afforded by Compton interactions in the detector. Polarization encodes information about the geometry of magnetic fields in gamma-ray sources and emission regions and adds a new observational pillar, this one in addition to the spatial/localization/structure, temporal and energy/spectral domains. The ability to provide gamma-ray polarimetric information will be crucial for a variety of investigations, including accretion processes in BH and SMBH systems, 
magnetic field structures in jets, and the emission mechanisms of GRBs. Polarization will also provide definitive insight into the presence of hadrons in extragalactic jets and the origin of UHECRs and very/extreme high energy cosmic neutrinos.

\section{Instrument and performances}

The payload of the e-ASTROGAM satellite (Fig. 5) consists of a single gamma-ray instrument operating over more than 4 orders of magnitude in energy $(\sim 150 \mathrm{keV}-3 \mathrm{GeV})$ by the joint detection of photons in both the Compton $(0.15-30 \mathrm{MeV})$ and pair $(>10 \mathrm{MeV})$ energy ranges. It is attached to a mechanical structure at a distance of about $90 \mathrm{~cm}$ from the top of the spacecraft platform, the space between the payload and the platform being used to: (i) host a time-of-flight (ToF) unit designed to discriminate between particles coming out from the telescope and those entering the instrument from below; (ii) host several units of the payload (the back-end electronics modules, the data handling unit, and the power supply unit) and (iii) accommodate two fixed radiators of the thermal control system, each of $5.8 \mathrm{~m}^{2}$ area. This design has the advantage of significantly reducing the instrument background due to prompt and delayed gamma-ray emissions from fast particle reactions with the platform materials. The e-ASTROGAM gamma-ray telescope is made up of three detection systems (Fig. 5): a silicon ( $\mathrm{Si}$ ) Tracker in which the cosmic gamma-rays undergo a Compton scattering or a pair conversion; a Calorimeter to absorb and measure the energy of the secondary particles and an Anticoincidence (AC) system to veto the prompt-reaction background induced by charged particles. It has a size of $120 \times 120 \times 78 \mathrm{~cm}^{3}$ and a mass of 1.2 tons (including maturity margins plus an additional margin of $20 \%$ at system level).

The Si Tracker comprises 5600 double-sided strip detectors (DSSDs) arranged in 56 layers. It is divided in four units of $5 \times 5$ DSSDs, the detectors being wire bonded strip to strip to form 2-D ladders. Each DSSD has a geometric area of $9.5 \times 9.5 \mathrm{~cm}^{2}$, a thickness of $500 \mu \mathrm{m}$, and a strip pitch of $240 \mu \mathrm{m}$. The total detection area amounts to $9025 \mathrm{~cm}^{2}$. Such a stacking of relatively thin detectors enables efficient tracking of the electrons and positrons produced by pair conversion, and of the recoil electrons produced by Compton scattering. The DSSD signals are read out by 860160 independent, ultra low-noise and low-power electronics channels with self-triggering capability. The Calorimeter is a pixelated detector made of a high- $Z$ scintillation material (Thallium activated Cesium Iodide) for efficient absorption of Compton scattered gamma-rays and electron-positron pairs. It consists of an array of 33856 parallelepiped bars of CsI(Tl) of $8 \mathrm{~cm}$ length and $5 \times 5 \mathrm{~mm}^{2}$ cross section, read out by silicon drift detectors (SDDs) at both ends, arranged in an array of 529 (= $23 \times 23$ ) elementary modules each containing 64 crystals. The depth of interaction in each crystal is measured from the difference of recorded scintillation signals at both ends. Accurately measuring the $3 \mathrm{D}$ position and deposited energy of each interaction is essential for a proper reconstruction of the Compton events. The Calorimeter thickness $(8 \mathrm{~cm}$ of $\mathrm{CsI}(\mathrm{Tl}))$ makes it a 4.3 radiation-length detector having an absorption probability of a $1-\mathrm{MeV}$ photon on-axis of $88 \%$. The third main detector of the e-ASTROGAM payload consists of an Anticoincidence system composed of two main parts: (1) a standard Anticoincidence, named Upper-AC, made of segmented panels of plastic scintillators covering the top and four lateral sides of the instrument, requiring a total active area of about $5.2 \mathrm{~m}^{2}$, and (2) a Time of Flight (ToF) system, aimed at rejecting the particle background produced by the platform. The Upper-AC detector is segmented in 33 plastic tiles ( 6 tiles per lateral 


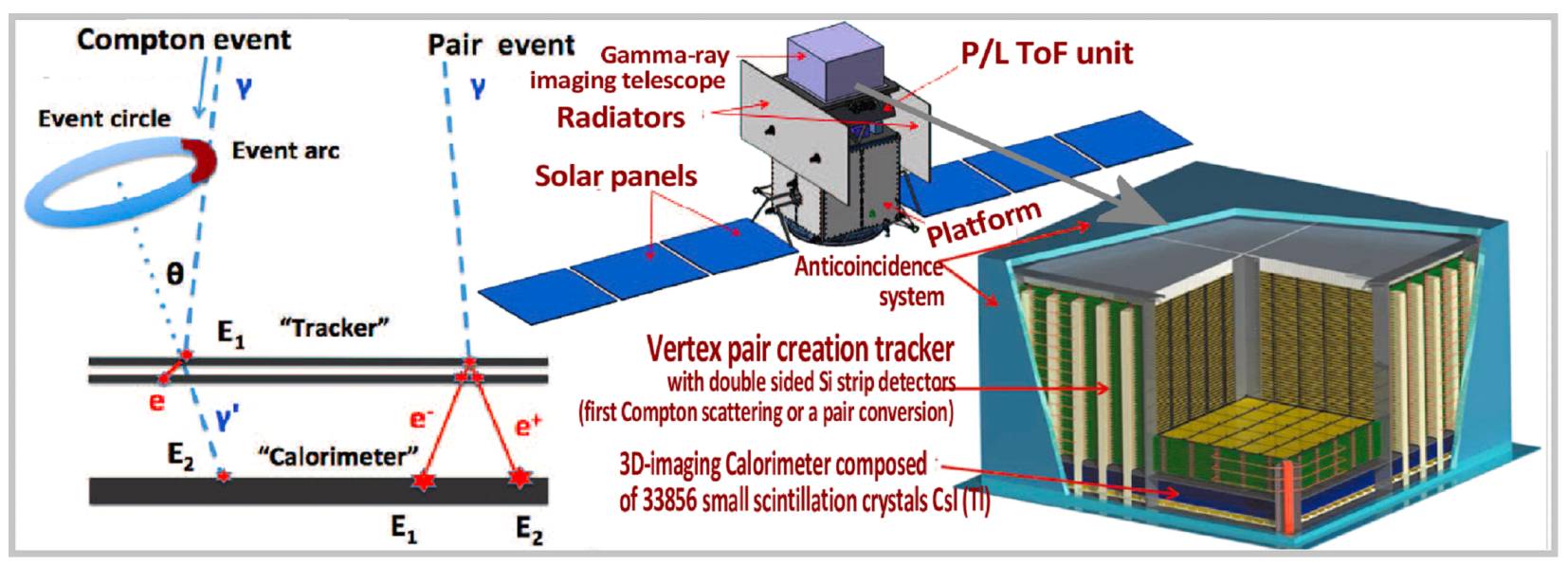

Figure 5: Schemes and drawings of the e-ASTROGAM spacecraft with solar panels deployed, the payload and the representative topologies for a Compton gamma-ray event and for a pair-conversion gamma-ray event in the tracker and calorimeter.

side and 9 tiles for the top) coupled to silicon photomultipliers (SiPM) by optical fibers. The bottom side of the instrument is protected by the ToF unit, which is composed of two plastic scintillator layers separated by $50 \mathrm{~cm}$, read out by SiPMs connected to Time Digital Converters. The required timing resolution is $300 \mathrm{ps}(1 \sigma)$.

For best environmental conditions, e-ASTROGAM it is thought to be launched into a quasiequatorial (inclination $i<2.5^{\circ}$ ) low-Earth orbit (LEO) at a typical altitude of about 550/600 km. The background environment in such an orbit is now well-known thanks to the Beppo-SAX and AGILE satellites. In addition, such a LEO is practically unaffected by precipitating particles originating from solar flares, a virtue for background rejection.

Extensive simulations of the instrument performances shows that e-ASTROGAM will achieve: - Broad energy coverage ( $\sim 0.15 \mathrm{MeV}$ to $3 \mathrm{GeV})$, with nearly 2 orders of magnitude improvement of the continuum sensitivity in the range $0.15-100 \mathrm{MeV}$ compared to previous missions (Fig. 1). - Excellent sensitivity for the detection of key gamma-ray lines e.g. sensitivity for the $847 \mathrm{keV}$ line from thermonuclear supernovae 70 times better than that of the INTEGRAL SPI.

- Unprecedented angular resolution both in the $\mathrm{MeV}$ domain and above a few hundreds of $\mathrm{MeV}$ i.e. improving the angular resolution of the CGRO COMPTEL telescope and that of the Fermi LAT instrument by a factor of $\sim 4$ at $5 \mathrm{MeV}$ and $1 \mathrm{GeV}$, respectively (e.g. the e-ASTROGAM Point Spread Function, $68 \%$ containment radius, at $1 \mathrm{GeV}$ is 9').

- Large field of view ( $>2.5$ steradians), ideal to detect transient Galactic and extragalactic sources, such as X-ray/gamma-ray binaries and GRBs.

- Timing accuracy of $1 \mu \mathrm{s}$ (at $3 \sigma$ ), ideal to study the physics of magnetars and rotation-powered pulsars, as well as the properties of terrestrial gamma-ray flashes.

- Pioneering MeV-GeV-energy polarimetric capability for both steady and transient sources [19].

\section{Gamma-ray polarimetry}

e-ASTROGAM has breakthrough capacity for gamma-ray polarimetry $[19,8]$ thanks to the fine 3D position resolution of both the Si Tracker and the Calorimeter, as well as the light mechanical structure of the Tracker, which is devoid of any heavy absorber material in the detection 

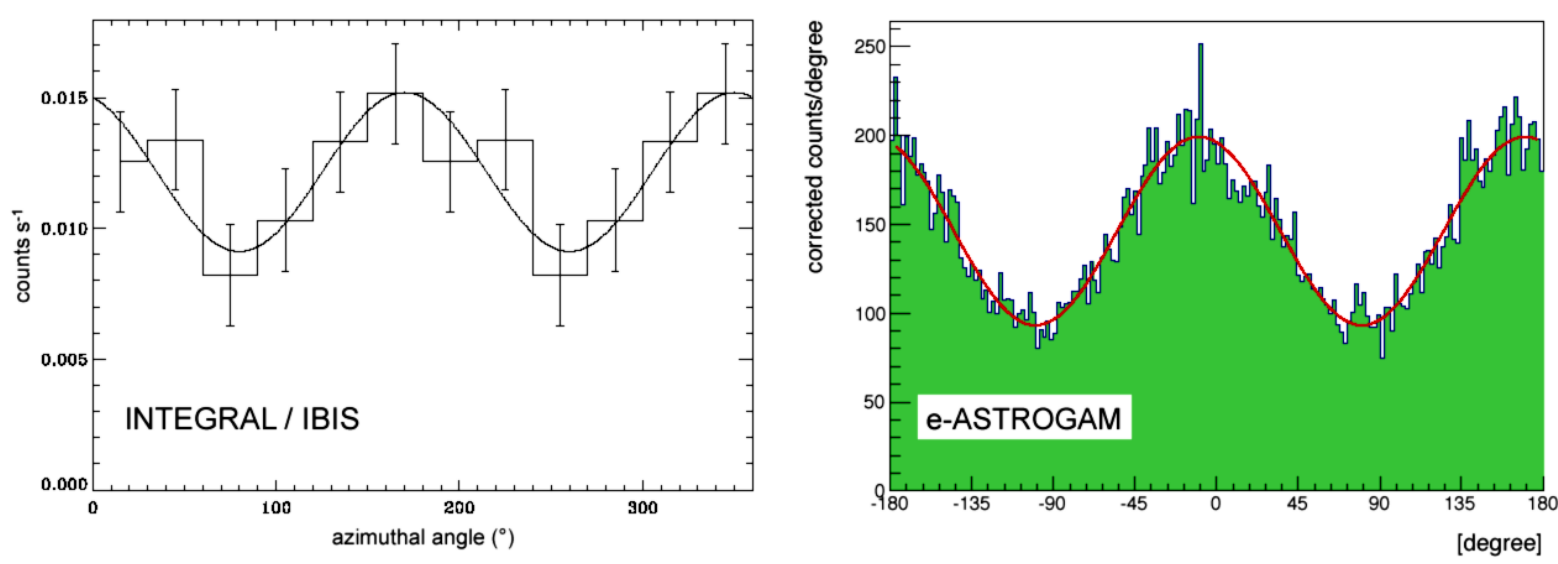

Figure 6: Left panel: Phase-averaged polarization diagram of the Crab pulsar and nebula obtained with INTEGRAL IBIS in the $300-450 \mathrm{keV}$ energy band by summing $1.831 \mathrm{Ms}$ of data taken between 2012 and 2014. The measured polarization fraction and angle are $\mathrm{PF}=(98 \pm 37) \%$ and $\mathrm{PA}=80^{\circ} \pm 12^{\circ}$, respectively. Right panel: simulation of the e-ASTROGAM polarization signal in the same energy band for an effective duration of observation of the Crab pulsar and nebula 100 times shorter than that accumulated by IBIS (i.e. $\left.T_{\mathrm{obs}}=1.831 \times 10^{4} \mathrm{~s} \simeq 5 \mathrm{~h}\right)$. The reconstructed polarization fraction and angle are $\mathrm{PF}=(98.0 \pm 2.4) \%$ and $\mathrm{PA}=80.2^{\circ} \pm 0.7^{\circ}$.

volume. The expected quality of the e-ASTROGAM polarization data compared to currently available observations of the Crab pulsar and nebula with INTEGRAL is reported in the example of Fig. 6. e-ASTROGAM would accumulate a much higher statistics than INTEGRAL IBIS in 100 times less exposure, and with a such excellent sensitivity and timing resolution, it will be able to provide a detailed gamma-ray view of the time-dependent gamma-ray polarization properties of the Crab nebula and pulsar, in particular during the cryptical periods of strong gamma-ray flaring activity, possibly associated with a totally new type of particle acceleration [3]. For example, a total exposure of $1 \mathrm{Ms}$ would allow a phase-resolved polarimetric study of the Crab pulsar with a time resolution of only $20 \mu \mathrm{s}$, which is $1 / 1650$ of the pulsar rotation period.

At low energies (0.2 - $2 \mathrm{MeV}$ ), e-ASTROGAM will achieve a Minimum Detectable Polarization (MDP) at the $99 \%$ confidence level of $20 \%$ for a $5 \mathrm{mCrab}$ source after one year of effective exposure. With such a performance, e-ASTROGAM will be able to study the polarimetric properties of many pulsars, magnetars, gamma-ray binaries [17], novae, microquasars and BH systems in the Galaxy. In the pair creation range, the measurement of polarization using the azimuthal orientation of the electron-positron plane is more challenging. Preliminary simulations show that a $\mathrm{MDP}_{99}$ of $\approx 20-40 \%$ in the energy range from 10 to $100 \mathrm{MeV}$ is within reach for bright gamma-ray sources such as the Vela and Crab pulsars.

Table 2 summarizes conservative estimates of the number of sources for which good polarization data are expected with e-ASTROGAM. The cumulative number of GRBs to be detected as a function of $\mathrm{MDP}_{99}$ was estimated from the simulated response of the gamma-ray instrument to linearly polarized GRBs at several off-axis angles in the range $\left[0^{\circ} ; 90^{\circ}\right]$. The GRB detection rate is expected to be $\sim 240$ per year, and a polarization fraction greater than $50 \%$ could be measured in $\sim 100$ GRBs per year. Such a high degree of linear polarization is consistent with the first GRB polarization results obtained by the Gamma-Ray Burst Polarimeter (GAP) aboard the IKAROS solar sail mission, and by INTEGRAL. Polarization information in GRBs can place strong new obser- 


\begin{tabular}{|l|c|c|}
\hline Type & Nb sources & Comments \\
\hline \hline GRBs & $\sim 300$ & Minimum detectable polarization of 10\%, 20\%, and \\
Blazars & $>17$ & 50\% in 16, 40, and 100 GRBs per year, respectively \\
& & 13 FSRQ and 4 BL Lacs (PKS 0716+714, PKS 2155-304, \\
Other AGN & $>6$ & Mkn 421 and Mkn 501) \\
& & Bright radio-quiet quasars and Seyfert galaxies: \\
Pulsars \& PWNe & $>18$ & 4151, IC4329a, NGC 4945, NGC 1068, 3C 120, Cen A \\
Magnetars & $>4$ & Persistent gamma-ray emission of 1RXS J1708-4009, \\
& & 4U 0142+61, 1E 1841-045, and SGR 1806-20 \\
$\gamma$-ray binaries & $>6$ & Currently known $\gamma$-ray binaries: PSR B1259-63, \\
& & LS I +61 303, LS 5039, HESS J0632+057, \\
Microquasars & $>9$ & Cyg X-1, Cyg X-3, GRS 1915+105, 1E1740.7-2942, SS 433, \\
& & GRS 1758-258, GX 339-4, XTE J1550-56, GRO J1655-40 \\
\hline
\end{tabular}

Table 2: Estimated number of sources of various classes whose polarization properties will be measured with e-ASTROGAM in the first three years of nominal mission.

vational constraints on the nature of the ultrarelativistic outflow, its geometry and magnetization, as well as on the high-energy radiation mechanisms; measurements of GRB polarization also have the potential to provide constraining limits on violation of Lorentz invariance [14].

Polarization measurements of blazars can provide crucial insight to the geometry of the emitting regions and allow to discriminate among various processes proposed as emission mechanisms. In particular, such measurements have the potential to identify the presence of hadrons in jets of BL Lac objects. e-ASTROGAM is expected to provide good polarization data for at least the 15 blazars already detected by COMPTEL and SPI, as well as for the two BL Lac objects PKS 2155304 and Mkn 501. However, with its unprecedented sensitivity in the $1-100 \mathrm{MeV}$ energy range, e-ASTROGAM should detect more than $~ 350$ blazars in three years, mostly MeV-peaked FSRQs, and good polarization data could be obtained for a significant fraction of them. It is important to note that MeV-peaked blazars are the most massive high redshift and jetted SMBHs in the Universe [12] and e-ASTROGAM is the only instrument capable to detect the most distant of them.

e-ASTROGAM is also expected to detect the gamma-ray polarization signal of a handful of Seyfert galaxies, and the measured polarization fraction and angle will provide important information on the geometry of the Comptonizing medium in these AGN (bright examples are the Seyfert 1 galaxies NGC 415 and IC 4329a, and the Seyfert 2 galaxies NGC 4945 and Cen A, all detected at $>200 \mathrm{keV}$ by INTEGRAL SPI, as well as the Compton thick AGN NGC 1068).

e-ASTROGAM will be able to study the polarimetric properties of the 18 currently known, rotation-powered, soft gamma-ray pulsars, which are thought to be significantly younger and more energetic than the pulsars detected in the GeV domain by Fermi LAT. Detailed phase-resolved polarimetry of the pulsed emission will be possible for a handful of bright MeV pulsars, such as PSR B0531+21 (Crab, see above), PSR B1509-58 (MSH 15-52) and PSR J1930-1852 (G54.1+0.3). The polarimetric measurements with e-ASTROGAM will shed new light on the processes of particle 
acceleration and pair cascades in the pulsar magnetosphere, as well as on the geometry and structure of the magnetic field in these systems. Magnetars are ultra-magnetized NS $\left(B \approx 10^{13}-10^{15} \mathrm{G}\right)$ powered by their own magnetic energy. e-ASTROGAM is expected to detect the gamma-ray polarization of both steady and flaring emissions from magnetars. Polarization measurements of the persistent soft gamma-ray emission of four magnetars will reveal the reprocessing of thermal photons emitted by the NS surface through resonant Compton scattering in the twisted magnetosphere. e-ASTROGAM should also provide invaluable information on NS physics by measuring the polarization of strong bursts from Soft Gamma Repeaters (SGR).

Gamma-ray binaries, of particular interest to understand accretion processes in astrophysical sources, are known to be powerful $\mathrm{MeV}$ emitters [11] and in the two scenarios describing the particle acceleration in these objects, (i) jets of a microquasar powered by accretion from a massive star, (ii) shocks between the relativistic wind of a young non-accreting pulsar and the wind of the massive stellar companion, the resulting nonthermal gamma-ray emission is expected to be polarized. e-ASTROGAM has the capability to measure the polarization degree of the six currently known gamma-ray binaries (Table 2). Gamma-ray polarimetry of microquasars with e-ASTROGAM is very promising as well. Simulated polarigrammes for e-ASTROGAM observations of Cyg X-1 in the "low" hard state show that the mission will be able to provide a detailed spectral and timeresolved gamma-ray view of the polarization properties of this system on a timescale of $10^{4} \mathrm{~s}$, thus answering questions regarding the different emitting media (Comptonized corona vs. synchrotronself Compton jets), while providing important clues on the composition, energetics and magnetic field of the jet. The excellent sensitivity of e-ASTROGAM in the MeV range will also give access to detailed studies of other, fainter, microquasars (like Cyg X-3, GRS 1915+105, 1E1740.7-2942), whose polarized gamma-ray emission is not currently detectable.

\section{5. e-ASTROGAM and the multimessenger astronomy}

e-ASTROGAM is uniquely positioned to be the space observatory of the 2030s with most complementarity for gravitational wave (GW) and cosmic neutrino astronomy.

The very large field of view of e-ASTROGAM (more than 1/5 of the sky for the Tracker and even larger for the Calorimeter in transient mode starting from $30 \mathrm{keV}$ ) will be essential to properly cover the GW error regions (bananas plots) and find electromagnetic (EM) counterparts to the GW events. The recent binary NS merger generating the GW event GW170817 and the corresponding short GRB detected by Fermi GBM and INTEGRAL $1.7 \mathrm{~s}$ after the GW signal has shown that the soft gamma-ray domain is the most appropriate band of the EM spectrum to identify the source and define the astrophysical context of the burst event [2]. In contrast, the number of optical transients spatially and temporally coincident with GW events is expected to be of the order of hundreds [16].

The on-axis prompt emission of GRBs associated to GW events shall be favorably detected with e-ASTROGAM . Advanced LIGO and Advanced Virgo can operate at design sensitivity, with an expected range for GW detection of $200 \mathrm{Mpc}$ for NS-NS mergers and $1 \mathrm{Gpc}$ for BH-NS systems. e-ASTROGAM will be able to quickly transmit spectral and positional information to the community to trigger the GRB follow-up by both ground-based and space facilities. In addition to serendipitous observations, e-ASTROGAM will be able to effectively point at sky regions with 

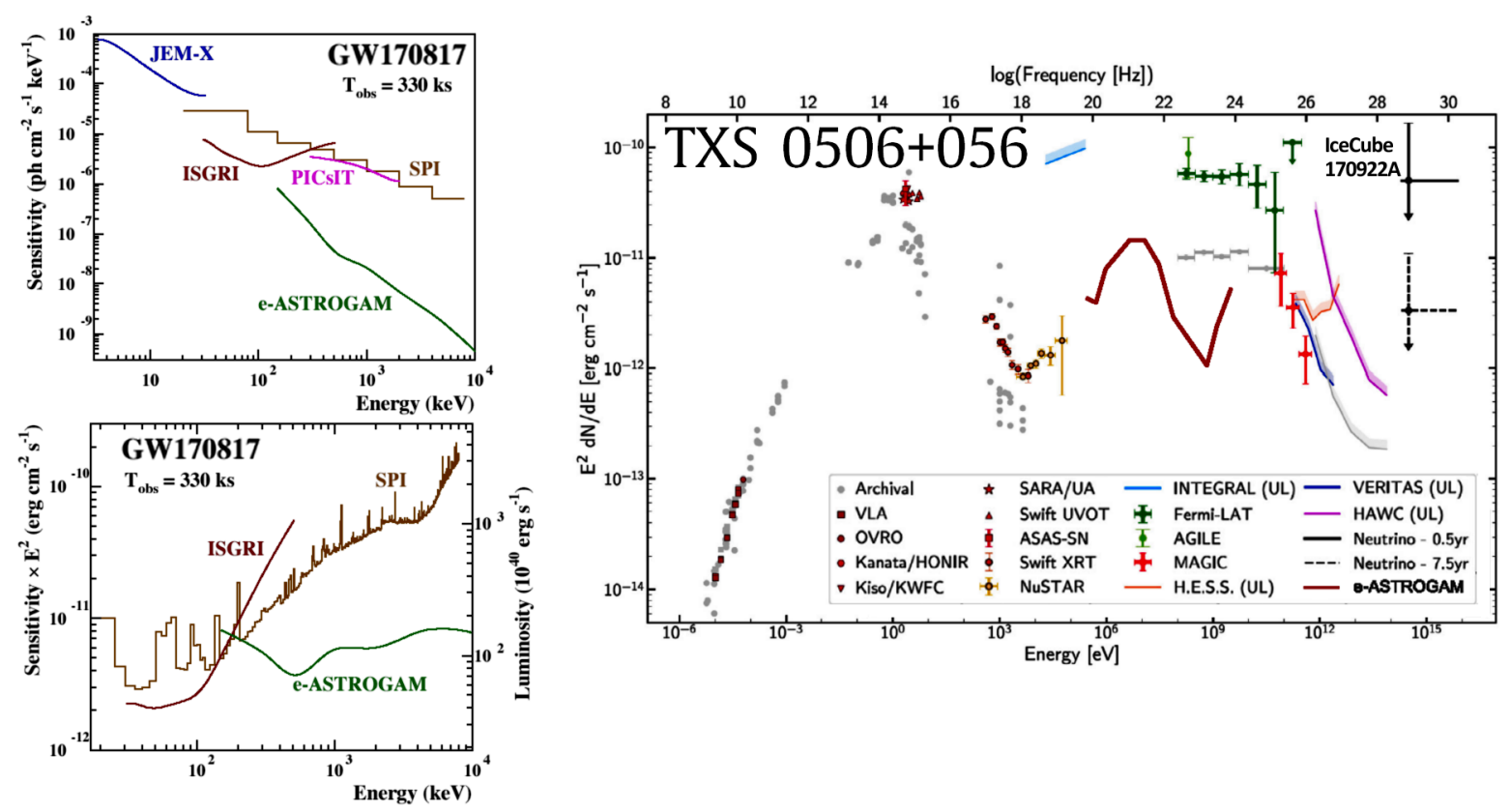

Figure 7: Left panels: continuum and narrow-line sensitivities reached in the INTEGRAL targeted followup observation of the gravitational wave burst GW170817 [2], compared to the corresponding sensitivities of e-ASTROGAM. All sensitivities are shown for a total exposure of $330 \mathrm{ks}$. The luminosity units of panel assume a distance to the source of $40 \mathrm{Mpc}$. Right panel: spectral energy distribution (SED) of the blazar TXS 0506+056. The green and red data points correspond to the gamma-ray flare observed by Fermi LAT and MAGIC of TXS 0506+056, associated to the IceCube neutrino detected event IC-170922A of about 290 $\mathrm{TeV}$ energy [1], with arrival direction consistent with the location of the blazar. Gray and black points are archival SED data. The e-ASTROGAM expected sensitivity line is calculated for an effective exposure of 7 days.

GW identifications, the mission requirement for the satellite repointing in case of a Target of Opportunity $(\mathrm{ToO})$ observation being within 6-12 hours, with the goal of reaching 3-6 hours.

e-ASTROGAM simulations based on the large GRB database of BATSE, Swift BAT, Fermi GBM yield detection rates of about 60 short GRBs and 180 long GRBs per year in the "Gammaray imager" trigger mode of the gamma-ray telescope (Table 1). Additional, softer bursts will be detected by the "Calorimeter burst search" mode of data acquisition (i.e. using triggers generated only by an increase of the Calorimeter count rate). The excellent sensitivity of e-ASTROGAM in the soft gamma-ray domain will also be crucial to reveal nearby off-axis GRBs associated to GW events.

The follow-up of GW170817 conducted at optical, infrared and ultra-violet wavelengths revealed the presence of an EM counterpart with emission consistent with a kilonova powered by the radioactive decay of r-process nuclei synthesized in the merger outflows [6]. e-ASTROGAM continuum and line sensitivities in the $\mathrm{MeV}$ range could allow to detect the radioactive decay gamma-rays from nearby kilonovae, thus directly probing the nucleosynthesis process thought to be responsible for the creation of approximately half the abundances of the nuclei heavier than iron. Figure 7 illustrates the superior sensitivity of e-ASTROGAM to detect the continuum and nuclear line emissions expected from the kilonova following a binary NS merger event like GW170817. The predicted gamma-ray line emission could be detected with e-ASTROGAM up to a maximum 
distance of $\sim 15 \mathrm{Mpc}$ [13]. e-ASTROGAM would coincide with the third generation of groundbased interferometric GW observatories, such as the Einstein Telescope and the Cosmic Explorer.

e-ASTROGAM could also be in operation at the same time as the Laser Interferometer Space Antenna (LISA), which will open GW observations to SMBHs expected to have magnetized circumbinary discs powering EM emission. Joint GW and gamma-ray detections by LISA and eASTROGAM could also come from binary systems of SMBHs like the gamma-ray blazars OJ 287 and PG 1553+113 [20, 5].

e-ASTROGAM sensitivity is adequate to measure the spectral energy distribution (SED) of neutrino sources detectable by IceCube, as one can see from the recent detection of the association of a 290-TeV neutrino event with an extended gamma-ray flare of the blazar TXS 0506+056 (Fig. 7 and [1]). Neutrinos are unique probes to study high-energy cosmic sources, since they are not absorbed by pair production via $\gamma \gamma$ interactions. Astrophysical high-energy neutrinos at $\mathrm{TeV}-\mathrm{PeV}$ energies are generated by the decay of charged pions produced in inelastic photo-hadronic $(p \gamma)$ or hadronuclear $(p p)$ processes, involving protons $\sim 20$ times more energetic than the resulting neutrinos. Photoproduction of neutrinos (and photons) via pion decay happens mainly via the $\Delta^{+}$ resonance slightly above kinematical threshold: $p \gamma \rightarrow \Delta^{+} \rightarrow N \pi$. The energy of the proton has to be $E_{p} \gtrsim 350 \mathrm{PeV} / \varepsilon$, where $\varepsilon$ is the target photon energy in eV. For UV photons, as expected in AGN jets, this translates into $E_{p} \gtrsim 10 \mathrm{PeV}$, i.e., above the knee: photoproduction of neutrinos on optical/UV photons is a likely indicator of UHECR acceleration. A simultaneous emission of hadronic gamma rays happens in both processes. An approximate relation holds at emission between the spectral production rates of neutrinos and gamma rays in hadronic production, and depending on the source optical depth, such photons are likely to further cascade, degrading their energy.

\section{Conclusion}

e-ASTROGAM is a concept for a gamma-ray space observatory that can revolutionize the astronomy of medium/high-energy gamma rays by increasing the number of known sources in this field by more than an order of magnitude and providing polarization information for many of these sources. e-ASTROGAM has breakthrough polarimetric sensitivity in $\mathrm{MeV}-\mathrm{GeV}$ gammaray band, that can be achieved for the first time. Thousands of sources are expected to be detected during the first 3 years of operations. Furthermore, the proposed wide-field gamma-ray observatory will play a major role in the development of time-domain, multi-wavelength and multi-messenger astronomy, providing valuable information for the localization and identification of gravitational wave sources. The e-ASTROGAM satellite project is thought to be complementary and crucial for multiwavelength investigations jointly done with radio (VLA, VLBI, ALMA, SKA), optical (JWST, E-ELT and other ground telescopes), X-ray (Athena) and TeV instruments (CTA, HAWC, LHAASO and other ground-based detectors). Special emphasis is given to fast reaction of eASTROGAM to transients and rapid communication of alerts. New opportunities in astro-particle physics (sources of gravitational waves, neutrinos, UHECRs) will be fully explored.

The $\mathrm{MeV}$ band is poorly explored but it is one of the richest energy domain for astrophysics, and the lack of comparable sensitivity observations in $\mathrm{MeV}$ regime hinders some progress in other wavebands. Indirect dark matter signal searches in $\mathrm{MeV}$ gamma ray regime can be very useful 
in the current post-WIMP era, where low-energy dark matter models and searches $(\mathrm{MeV}, \mathrm{keV}$ down to feV masses) are growing in importance. To be remarked also that $\mathrm{MeV}$ astronomy is for nuclear processes and nucleosynthesis what optical/IR astronomy is for atomic transitions and a general-purpose satellite like e-ASTROGAM is able to serve broad and different astronomical communities, with a likely large return in science results and publications.

Despite the unfavorable outcome for this mission by ESA (not selected in M4 and M5 calls, 2014, 2016), there is a broad international interest and rising attention and concern for the problem of leaving the Universe, and the multi-messenger phase-space, unobserved and unattended in a such wide window of the electromagnetic spectrum. An empty window spanning more than 6 decades in photon energy, placed between Athena and CTA. Institutes and researcher in 30 countries have collaborated in the e-ASTROGAM project for the last M5 call, and among these, 19 are European nations. The e-ASTROGAM instrument is based on an innovative design, which minimizes any passive material in the detector volume, and performances have been assessed through detailed simulations using state-of-the-art tools. The results fully meet the scientific requirements of the proposed mission. One possible future for this project could be, to find substantial interest in the scientific community of the Russian Federation and interest and a substantial funding by ROSCOSMOS that could contribute (lead too) the project, joined with other national space agencies and institutes in Europe, addressing the construction and launch of this MeV satellite. This following the successful footsteps of space astronomical telescopes like Pamela and INTEGRAL. Another possibility may be the realization of a similar MeV space telescope in USA, like is represented by the current AMEGO probe mission concept [15]. There is not any $\mathrm{MeV}$ satellite accepted by any agency, thus a productive and synergetic effort with the right mix of competition (during the study and design phase with a couple of different projects one American and one Russian+European, for example) and collaboration (when one satellite will be selected at last all the efforts and collaborations are expected to merge) may substantially help to accelerate and move forward the current worrying situation, toward the construction of one selected satellite to be launched at the end of 2020s.

\section{References}

[1] Aartsen, M. G., Ackermann, M., Adams, J. et al. 2018, Science, 361, eaat1378

[2] Abbott, B. P., Abbott, R., Abbott, T. D., et al. 2017, ApJL, 848, L13

[3] Abdo, A. A., et al. 2011, Science, 331, 739

[4] Abdollahi, S., et al. 2020, ApJS, accepted

[5] Ackermann, M., et al. 2015, ApJ, 813, L41

[6] Arcavi, I., et al. 2017, Nature 551, 64

[7] Churazov E. et al. 2014, Nature, 512, 406

[8] De Angelis A. et al. 2018, Journ. High Energy Astroph., 19, 1 (arXiv:1711.01265)

[9] De Angelis A. et al. 2017, Experimental Astron. 44, 25-82

[10] Diehl R. et al. 2006, A\&A, 439, 45

[11] Dubus, G., 2013, A\&A Review 21, 64

[12] Ghisellini G. et al. 2010, MNRAS, 405, 387

[13] Hotokezaka, K., et al. 2016, MNRAS, 459, 35 
[14] Laurent, P., et al. 2011, Science, 332, 438

[15] McEnery, J., et al. 2019, Astro2020 APC White Paper, (arXiv:1907.07558)

[16] Nissanke, S., et al. 2013, ApJ 767, 124

[17] Siegert T. et al. 2016, Nature, 531, 341

[18] Tatischeff V. et al. 2018a, Proc. SPIE, 10699, 106992J

[19] Tatischeff V. et al. 2018b, Journ. Astron. Tel., Inst. Syst. 4, 011003

[20] Valtonen, M. J., et al. 2016, ApJ, 819, L37

\section{DISCUSSION}

JORDI ISERN: To be fully useful for supernova (SN) research e-ASTROGAM should have a good sensitivity below $300 \mathrm{keV}$, for example to detect the $158 \mathrm{KeV}{ }^{56} \mathrm{Ni}$ line, or there will be a substantial loss of interest in this topic. To be considered INTEGRAL waited 12 years since the launch, to detect the first gamma-ray lines from a Type Ia supernova (with SN 2014J).

STEFANO CIPRINI: After about three weeks from the supernova explosion time, gamma-ray spectral models predicts strong $158 \mathrm{keV}, 750 \mathrm{keV} 812 \mathrm{keV}{ }^{56} \mathrm{Ni}$ lines and an intense $847 \mathrm{keV}{ }^{56} \mathrm{Co}$ line, given by the freshly synthesized radioactive ${ }^{56} \mathrm{Ni}$ in the outermost layers of the supernova ejecta. All can be observed in case of nearby, bright, supernova being in the energy range of the e-ASTROGAM detector. Its has a nominal energy range from $0.3 \mathrm{MeV}$ to $3 \mathrm{GeV}$, with onetwo orders of magnitude improvement in continuum sensitivity in this range compared to previous instruments. The lower energy limit can be pushed to energies as low as $150 \mathrm{keV}$, albeit with rapidly degrading of angular resolution, for the tracker. Furthermore is possible to lower the threshold down to energies as low as $30 \mathrm{keV}$ for gamma-ray bursts (and bright transients like potential very nearby SNs) detection with the calorimeter. Specifically simulations show that for a SN event like SN 2014J (distance of $3.5 \mathrm{Mpc}$ ) the spectrum after 70 days from the explosion is dominated by ${ }^{56} \mathrm{Co}$ lines, scattered continuum and annihilation emission. Such event would be detected at $300 \sigma$ by e-ASTROGAM . Such simulations also show that extremely bright lines of radioactive ${ }^{56} \mathrm{Ni}$ at $158 \mathrm{kev}$ and $810 \mathrm{keV}$ can be clearly being detected in the spectrum by e-ASTROGAM with a 500ks exposure at 17.5 days after the SN explosion. Believing in the occurrence of a nearby SN in the first three years of mission e-ASTROGAM is therefore also a full gamma-ray "kilonova/supernova telescope".

YURI T. YURKIN: while pair conversion events have obvious and efficient triggers, I doubt that cosmic gamma-rays that undergo a Compton scattering can have a good trigger, also because of the lack of a clear conversion and track in the detectors.

STEFANO CIPRINI: the Si Tracker comprises 5600 double-sided strip detectors (DSSDs) arranged in 56 layers, divided in four units of $5 \times 5$ DSSDs, with detectors being wire bonded strip to strip to form 2-D ladders (total detection area of $9025 \mathrm{~cm}^{2}$ ). Such a stacking of relatively thin 
detectors enables both efficient tracking of the electrons and positrons produced by pair conversion, and of the recoil electrons produced by Compton scattering. Hence a first fact is the expected efficient tracking of the recoil electrons for the Compton regime. The DSSD signals are read out by 860160 independent, ultra low-noise and low-power electronics channels, and have self-triggering capability. The Calorimeter is a pixelated detector made of a high-Z scintillation material (Thallium activated Cesium Iodide) for efficient absorption of Compton scattered gamma-rays and electronpositron pairs, with interaction depth in each crystal measured from the difference of recorded scintillation signals at both ends. Accurately measuring the 3D position and deposited energy of each interaction is essential for a proper reconstruction of the Compton events. Trigger for Compton tracked events is challenging but several baseline trigger option are possible and under study (also hardware prototypes for real-data studies like baloon flights, beam tests in accelerators, micro/cube satellite are being built and planned to study the best configuration and also the problem of triggers and background in Compton regime). A trigger example is a five-fold coincidence of the hits in two consecutive Si planes and the presence of a signal from the calorimeter, with no veto signal in the ACD within the triggering time window of a few microseconds. The requirement of hits in two consecutive Si planes assumes the detection of the scattered electron for Compton events, and the trigger can be re-configured to require a hit in only one Si plane and in the calorimeter, a configuration allowing for the detection also of "untracked" Compton events. All the background components were carefully taken into account in the sensitivity assessment, including the cosmic diffuse gamma-ray background, the atmospheric gamma-ray emission, the reactions induced by albedo neutrons, and the background produced by the radioactivity of the satellite material activated by solar-wind/cosmic fast protons and alpha particles. Several trigger options were studied by detailed numerical simulations to evaluate the efficiency of background rejection. These simulations have shown that the e-ASTROGAM sensitivity at $1 \mathrm{MeV}$, i.e. in full Compton scattering regime, will be better than that of the previous missions by about two orders of magnitude. 